\title{
Clinical trials in urological oncology: COVID-19 and the potential need for a new perspective
}

\author{
Jiasian Teh ${ }^{1,2}$ (1) Ellen O'Connor ${ }^{1,2}$ - Jasamine Coles-Black ${ }^{2}$. Nathan Lawrentschuk ${ }^{1,3,4}$
}

Received: 1 June 2020 / Accepted: 14 August 2020 / Published online: 4 September 2020

○) Springer-Verlag GmbH Germany, part of Springer Nature 2020

\begin{abstract}
The COVID-19 pandemic has led to the suspension, termination or alteration of thousands of clinical trials as the health emergency escalated globally. Whilst the rapid suspension of certain clinical trials was necessary to ensure the safety of high-risk or vulnerable trial participants as well as healthcare workers, the long-term ramifications that this delay will have on the field of urologic oncology is unknown. The COVID-19 pandemic has highlighted the need to plan for and implement new strategies to advance our understanding of unmet areas of need in urologic oncology. The COVID-19 pandemic has led to the suspension, termination or alteration of thousands of clinical trials as the health emergency escalated globally. Whilst the rapid suspension of certain clinical trials was necessary to ensure the safety of high-risk or vulnerable trial participants as well as healthcare workers, the long-term ramifications that this delay will have on the field of urologic oncology is unknown. The COVID-19 pandemic has highlighted the need to plan for and implement new strategies to advance our understanding of unmet areas of need in urologic oncology.
\end{abstract}

Keywords COVID-19 · Urologic oncology $\cdot$ Clinical trial

New therapeutic options in urological oncology are highly sought after with many randomised control trials (RCT) established to address areas of unmet need in recurrent and metastatic prostate cancer, non-muscle invasive and metastatic bladder cancers and rare urological malignancies such as penile cancer. However, the COVID-19 pandemic has led to 10,174 clinical trials registered with the US government site ClinicalTrials.gov being suspended, terminated, withdrawn or moved to 'not recruiting' status in March and April 2020 as the health emergency escalated globally [1]. Whilst the rapid suspension of certain clinical trials was necessary to ensure the safety of high-risk or vulnerable trial

Jiasian Teh

jiasian@gmail.com

1 Division of Cancer Surgery, Peter MacCallum Centre, Melbourne, VIC, Australia

2 Department of Surgery, Austin Hospital, University of Melbourne, Melbourne, VIC, Australia

3 Department of Surgery, Department of Urology, University of Melbourne and Royal Melbourne Hospital, Melbourne, VIC, Australia

4 EJ Whitten Prostate Cancer Research Centre at Epworth Healthcare, Melbourne, VIC, Australia participants and preserve resources during the COVID-19 pandemic, there are ethical considerations to delaying access to potentially successful treatments. While the potential long-term ramifications of the postponement of clinical trials may have is unknown, it may be far-reaching in terms of patient outcomes, data interpretation and drug development, with the full impact yet be realised $[2,3]$.

Randomised control trials (RCTs) are accepted as the only clinical experimental study design that enables strong inference of causality and provides the reference standard for the evaluation of new treatment modalities. However, this traditional approach used to generate strong clinical evidence is a slow and expensive process, with many such trials ultimately identifying little or no difference in benefit of the investigated treatment [4]. Novel clinical trial designs, such as multi-arm, multistage, basket studies and tumour-agnostic approaches are emerging as a robust way to advance innovation in urological oncology. In addition, big data sources such as electronic health records, administrative databases and platforms such as the Cancer Genome Atlas Program, which hosts the open-source collection and analysis of the molecular characterization of thousands of tumours and matched normal samples, will be invaluable in minimizing research waste and efficiently 
identifying solutions to unmet areas of need in urology cancer care [5].

New targets in urological oncology are emerging rapidly, owing in part to the advances in the molecular and genomic characterisation of tumours afforded by nextgeneration sequencing techniques, which have led to the understanding that urological cancers are a heterogenous population, even within specific tumour types [6]. Targeted molecular therapies and improved access to point of care genomic testing brought about by the decreasing costs associated with next-generation sequencing is shifting the traditional paradigm of standardized treatment for each cancer histological subtype to individualised approaches at a molecular level, so long as the individual patient has the target alteration in question. These tumour agnostic approaches have been approved for use since the Food and Drug Administration (FDA) accelerated approval for pembrolizumab, an anti-programmed cell death-1 (PD-1) monoclonal antibody (mAb), in 2017 for use in the treatment of patients with unresectable or metastatic solid tumours that were identified as having microsatellite instabilityhigh (MSI-H) or deficient DNA mismatch repair [7]. These novel approaches to treating urological malignancies must be considered in the post-COVID-19 pandemic clinical environment as delays in the reporting of results from existing trials may leave many patients with advanced or complex disease without further treatment options.

The challenges presented by the COVID-19 pandemic have been extensive with impacts on the administration of clinical care and research at every level, including impaired access to the healthcare system, travel restrictions and patient hesitancy to participate in trials. As a true testament to the scientific spirit, there are already 1409 COVID-19 related clinical trials registered on ClinicalTrials.gov [1]. However, this demonstrates a shift in resources away from oncology and a further exacerbation of the "orphan" status of rare cancers. In addition, the impact on the private biotech sector, which is reducing the number of early stage clinical trials and delaying the start of new trials, will undoubtedly have an impact on the drug discovery pipeline. Many companies have prioritised COVID-19-related research and have channelled resources away from oncology. The delay and interruption of clinical trials may also lead to difficulties within the private sector in sourcing significant additional funding in a volatile economic climate. Sponsors will need to balance patient safety, trial integrity, and statistical power considerations against funding and revenue limitations. The ability to rapidly adapt in a shifting environment will be key to minimizing disruptions to delivering much needed advancements in oncology. Finally, as different states and countries begin the heterogenous road to recovery, well-considered strategies as to how clinical trials spanning multiple sites should be reinstated are needed.
The COVID-19 pandemic has highlighted the need to plan for and implement new strategies to advance our understanding of unmet areas of need in urologic oncology. These can only be answered with attentive trial design and transparent reporting. In a climate of a global pandemic, decreased research funding and increased scrutiny by policymakers and the general public of health-care expenditures, it is essential that urologists remain at the forefront of medical advancement and improving patient care by becoming familiar with innovations in trial design and reporting practices. The rapid adoption and implementation of novel trial designs has been expedited by the current COVID-19 pandemic. However, there are important lessons to be learnt about the structure of trials to allow a focus on minimizing research waste by asking thoughtful and specific questions, conducting trials within areas of existing guideline deficiency and using standard outcome sets that incorporates measures important to patients and the rapidly changing face of clinical practice. This provides an opportunity for trials to be streamlined, whilst maintaining patient centred outcomes at the forefront, and an opportunity to look at minimizing barriers to traditional formats of oncology research.

Author contributions JT: protocol/project development, data collection, manuscript writing/editing. EOC: manuscript writing/editing. JCB: manuscript writing/editing. NL: protocol development, manuscript writing/editing.

\section{Compliance with ethical standards}

Conflict of interest The authors declare that they have no conflict of interest.

Ethical approval This research did not involve human or animal participants and as such no ethics approval was required.

Research data policy Authors are responsible for correctness of the statements provided in the manuscript. See also Authorship Principles. The Editor-in-Chief reserves the right to reject submissions that do not meet the guidelines described in this section.

\section{References}

1. U.S. National Library of Medicine. ClinicalTrials.gov 2020. https ://clinicaltrials.gov/ct2/home. Accessed 13 May 2020

2. Chen R, Liang W, Jiang M, Guan W, Zhan C, Wang T et al (2020) Risk factors of fatal outcome in hospitalized subjects with coronavirus disease 2019 from a nationwide analysis in China. Chest. https://doi.org/10.1016/j.chest.2020.04.010

3. Gostin LO, Friedman EA, Wetter SA (2020) Responding to Covid19: how to navigate a public health emergency legally and ethically. Hastings Cent Rep 50(2):8-12 
4. Parmar MKB, Barthel FMS, Sydes M, Langley R, Kaplan R, Eisenhauer E et al (2008) Speeding up the evaluation of new agents in cancer. J Natl Cancer Inst 100(17):1204-1214

5. Institute NC. The Cancer Genome Atlas Program 2020. https:// www.cancer.gov/about-nci/organization/ccg/research/structural -genomics/tcga. Accessed 13 May 2020

6. Chalmers ZR, Connelly CF, Fabrizio D, Gay L, Ali SM, Ennis R et al (2017) Analysis of 100,000 human cancer genomes reveals the landscape of tumor mutational burden. Genome Med 9(1):34
7. Le DT, Durham JN, Smith KN, Wang H, Bartlett BR, Aulakh LK et al (2017) Mismatch repair deficiency predicts response of solid tumors to PD-1 blockade. Science 357(6349):409

Publisher's Note Springer Nature remains neutral with regard to jurisdictional claims in published maps and institutional affiliations. 